\title{
Philosophiques
}

\section{Une alternance à l'exclusion : la dia-logie}

\section{Jacques-Bernard Roumanes}

Volume 11, numéro 2, octobre 1984

Égalité, justice et différence

URI : https://id.erudit.org/iderudit/203263ar

DOI : https://doi.org/10.7202/203263ar

Aller au sommaire du numéro

Éditeur(s)

Société de philosophie du Québec

ISSN

0316-2923 (imprimé)

1492-1391 (numérique)

Découvrir la revue

Citer cet article

Roumanes, J.-B. (1984). Une alternance à l'exclusion : la dia-logie.

Philosophiques, 11(2), 353-372. https://doi.org/10.7202/203263ar

\section{Résumé de l'article}

L'individualisme exclusionniste versus le collectivisme massificateur, tel est le non-choix envers et contre lequel tente de se formaliser la problématique des théories de l'égalité, dans la perspective des rapports hommes-femmes. Face à cette signalée impasse, la dia-logie, avec son économie d'alternance, ouvre à une resémantisation de la dialectique et du monologisme qui la fonde. d'utilisation que vous pouvez consulter en ligne.

https://apropos.erudit.org/fr/usagers/politique-dutilisation/ 


\title{
UNE ALTERNATIVE À L'EXCLUSION : LA DIA-LOGIE*
}

\author{
par Jacques-Bernard Roumanes
}

\begin{abstract}
RÉSUMÉ. L'individualisme exclusionniste versus le collectivisme massificateur, tel est le non-choix envers et contre lequel tente de se formaliser la problématique des théories de l'égalité, dans la perspective des rapports hommes-femmes. Face à cette signalée impasse, la dia-logie, avec son économie d'alternance, ouvre à une resémantisation de la dialectique et du monologisme qui la fonde.
\end{abstract}

ABSTRACT. Exclusionist individualism versus massifying collectivism, such is the non-choice against which the theories of equality in men-women relationships attempt to be formalised. Before this deadlock, dia-logie with its principle of alternation, bestows a new meaning upon dialectics and the monologism upon which it is based.

« Tous les massacres ont été accomplis par vertu, au nom de la religion vraie, du nationalisme légitime, de la politique idoine, de l'idéologie juste; bref au nom du combat contre la vérité de l'autre . . . "

François Jacob ${ }^{1}$

\section{UNE ALTERNATIVE À L'EXCLUSION . . .}

Fondée sur le principe d'exclusion - annexion de l'autre par l'un, la dynamique du système institutionnel apparaît à l'analyse d'une simplicité dérisoirement extrême : tout ramener au " même ». Tout, c'est-à-dire le monde complexe, les choses multiples et les (autres) êtres innombrables de ce monde à l'unité,

\footnotetext{
* Cet article utilise une économie répétitive dans le but d'éclairer au mieux l'orientation conceptuelle de certains contenus. On ne s'étonnera donc pas de retrouver en cours de développement la même idée déployée selon divers aspects de significations.
}

1. F. Jacob, le jeu des possibles, Paris, éd. du Seuil. 1981, p. 12. 
à l'identité, à la même chose, à la même loi, à un seul Ordre, à un seul mot d'ordre, à un unique pouvoir : à un Centre Originaire...

À l'inverse de l'univocité réifiante du principe d'exclusion, on observe la dynamique d'un échange alternatif des partenaires réels, où le pouvoir n'est plus un monopole exclusif, mais un trafic incessant, un légitime service de voirie, de police et de communication purement fonctionnel. Ce qu'il prétend être d'ailleurs, selon le slogan que résume le seul mot de : démo/ cratie. Il s'agit donc pour nous, à partir de ces points, de théoriser sur un certain nombre de notions pouvant montrer le bienfondé de préférer à ce principe d'exclusion un principe d'alternance. Ou plutôt, puisqu'ils sont déjà antagonistes dans la culture, de redonner la prépondérance au dernier, comme plus économique et plus réaliste.

$$
* *
$$

Moi, le fils de millions de pères, je serai le père de millions de fils, dit superbement le poète. Et, pas plus pour procréer que simplement survivre ou même exister, il ne nous paraît pas pensable d'envisager, surtout logiquement, de se passer, de s'abstraire les uns des autres. Nous évoluons dans un monde qui s'impose à nous comme nécessairement commun quant à nos aspirations et besoins particuliers. Envisagée sous cet angle, toute prétention à l'exclusion et à l'univocité devrait apparaître conduisant à la mort ou à l'aliénation - comme étant, radicalement, impossible ; comme s'opposant fondamentalement à la possibilité même de vivre.

Or, prendre le pouvoir, c'est précisément opérer stratégiquement, mythologiquement, institutionnellement, rationnellement ou autrement . . ., cette exclusion ou cette annexion « impossible » des autres par les uns; et de manière telle que les uns, seuls, en tirent univoquement bénéfice. Bref, si une telle chose demeure impossible que de m'exclure de mon corps (de femme, par exemple) pour se l'annexer " réellement ", il n'en va pas de même au plan dénotatif du champ culturel. Là, l'étrange, l'impensable, l'impossible exclusion a bel et bien lieu qui m'aliène (comme femme) au corps social. 
Tel est le rapport classique qui unit l'esclave et son maître, d'une manière générale le dominé au dominant et, traditionnellement encore de nos jours, sauf exceptions géographiquement restreintes la femme à l'homme, mais aussi l'homme à l'homme.

Par « impossible » nous voulons aussi signifier qu'une telle exclusion-annexion, légale, ne correspond dans les faits, que cataphoriquement ${ }^{2}$, au pays réel des hommes et des femmes (réels) dans leur rapport de société. Ce pays légal, que dessine schématiquement la trame des institutions ne recouvrant effectivement qu'un réseau relationnel particulier, lequel en définitive, se résume pour l'essentiel, aux (inévitables) interdictions de violence dans la relation quotidienne des partenaires du jeu social. Mais les rapports non extrêmes et non violents, donc non légaux, par leur variété, leur nombre, et par le fait qu'ils tissent la trame même des relations sociales, sont de loin les plus importants.

Entre la bouche et l'oreille, c'est-à-dire entre le commerçant et son client, entre le présentateur et le téléspectateur, entre l'homme politique et l'électeur, entre chacun des individus des deux sexes dans la rue, au travail comme en vacances, entre le mari et la femme, entre le couple et leurs enfants . . . les rapports sont trop aléatoires, le jeu des dispositions tellement illimité, instable, fluctuant et même le développement de la relation trop sûrement imprévisible. Aussi le rapport le plus économique demeure encore, non pas celui imposé par la loi, mais bien l'irrationnel contrat de confiance. Confiance toute relative certes, aveuglément limité peut-être, mais délégation inévitable de soi à autrui, toujours agissante entre le patron et l'employé comme jadis entre le maître et l'esclave. Tant il s'avère impossible d'envisager pouvoir tout vérifier, mesurer, contrôler, dominer . . . Car il faut une indispensable dose d'acceptation, une frange de consentement mutuel et de volonté de réciprocité, pour que ce rapport dominant/dominé puisse s'établir, mais sur-

2. Cataphoriquement, de cataphore (étym. ce qui est porté par le haut, fait d'en-haut), néologisme qui veut signifier que, dans la relation légale, c'est la loi qui, délimitant le champ du possible est donnée pour constituer les individus (en sujets) et par là a primauté sur eux. En d'autres termes, c'est soutenir que : la carte est le fondement du territoire qu'elle balise symboliquement. 
tout durer comme il dure. Partout et depuis si longtemps . . . et même tel qu'il ressurgit sous l'impact phantasmạtique du rêve totalitaire des cybernéticiens du meilleur des mondes.

C'est une lecture lucide et minutieuse de cet « autre » rapport, ce rapport de réciprocité constitutive, permettant aux partenaires de s'échanger pour l'immense majorité de leurs actions et de leurs paroles, qui peut nous introduire au moyen d'articuler cette alternative à l'exclusion que nous nommons : dia-logie (le mot "dialectique » ayant été écarté comme trop lourdement hypothéqué). En ayant soin de préciser que, si cette relation colloquiale n'était pas absolument vitale et formellement indispensable, notre perspective deviendrait vite illusoire. Tandis que, fondée sur l'essentiel des paroles et des actes - inaperçus parce que non institutionnalisables, non légaux ou illégaux - du jeu de société, elle s'offre à nos investigations comme le continent noir par excellence, à explorer.

La question se pose alors : hommes et femmes, peut-on exercer notre pouvoir, tous, chacun, en même temps, les uns et les autres ? Autrement que les uns sur les autres ? c'est-àdire par voie d'exclusion des uns par les autres? Stricto sensu, en termes éthiques et politiques, une démocratie qui ne soit pas une anarchie est-elle possible ? Corrélativement à la problématique théorique de l'égalité, cette démocratie a-t-elle encore quelque visage heuristique et pratique à nous montrer qui ne soit pas cynique ou simplement utopique (au sens d'Habermas)?

$$
\text { ** }
$$

En premier lieu, on doit faire remarquer que, traditionnellement, les énoncés théoriques ramènent invariablement les termes examinés (ex. le sujet masculin vs le sujet féminin) à un positionnement prétendu «fondationnel », par rapport à eux, à cause de la médiation qu'ils opèrent entre ces termes. Pour cette raison, ces énoncés (ex. la loi, les contrats sociaux) sont systématiquement posés par le théoricien (ex. le législateur) comme " au dessus » des termes qu'ils permettent de distribuer. En bref, le contrat social reste toujours hors d'atteinte des personnes qui deviennent les otages de sa référence. Le propre de tels énoncés, dits de vérité, qu'ils soient d'ordre juridique, méta- 
physique ou scientifique - outre la puissance exorbitante dont ils dotent politiquement le définisseur, ou le groupe qui les énonce, au détriment relatif de ceux qui les adoptent ou sont "forcés » de les suivre - est de constituer un corps de référence absolument stable. Ou bien encore une axiomatique fondationnelle, en ce sens que leur position (non pas leur contenu), supra ou méta rationnelle, ne peut pas être mise en cause. Et cela, suivant les dispositions même du contrat de raison passé entre les individus sujets à son application.

Il y a un cercle vicieux dont il nous semble qu'une problématique envisageant de traiter des théories de l'égalité devrait d'abord et avant toute chose tenter de se dégager, pour précisément examiner d'un autre point de vue l'égalité en question. Car il serait paradoxal de chercher à renouveler une telle question en la reposant dans la perspective même qui a suscité le problème. C'est-à-dire la position classique qui, a priori ou a posteriori exclut toute relation dia-logique, au profit d'une position logique univoque et hiérarchique. Le jeu de proximité des partenaires, et les partenaires eux-mêmes sont annexés ou exclus comme abstractions purement référentielles d'une axiomatique théorique (par exemple, telle métaphysique, tel darwinisme; tel féminisme . . . à l'exclusion de tous les autres). Celle-ci ne saurait être qu'inévitablement manipulée par le groupe qui, selon nous, le promeut comme justification rationnelle pour privilégier ses intérêts et sa praxis. A-t-il jamais existé une seule théorie mise de l'avant par un seul groupe, et qui propose comme programme : l'assujettissement et la destruction réels de ce groupe et des membres qui le composent ${ }^{3}$ ?

Quel peut donc être cet « autre point de vue » qui ne soit pas un : ailleurs, une vérité, un tout-autre, une axiomatique évidente ou absolue, etc. en dehors de la question, et qui loin de réfuter ou de négliger le précieux apport que constitue, quoiqu'on le critique, cet irremplaçable outillage conceptuel, cherchera à l'utiliser au mieux ? Mais non plus en s'assujettissant à sa médiation, mais au contraire en le subordonnant à ses fins propres.

3. C'est-à-dire au delà des slogans idéologiques à visées proprement stratégiques ; comme par exemple l'utopie marxiste de la destruction de l'État et de sa bureaucratie dans la perspective du Grand soir. 
Cet autre point de vue, c'est précisément celui de "l'autre ". C'est-à-dire celui d'une altérité envisagée comme irréductible au discours de la connaissance vraie ; contrairement à la dialectique classique qui tente inexorablement d'identifier l'un et l'autre (chacun des partenaires), au même. Ce point de vue de l'autre, pour reprendre l'exemple juridique et politique qui accompagne ce développement, c'est supposer le point de vue d'un : sujet légal de type féminin, qui ne serait pas « nécessairement " la métaphore du sujet légal de type masculin. Lequel sujet féminin, par la structure même de la distribution logique institutionnelle, n'aura jamais, c'est à craindre, d'autre avenir que de lui être «naturellement "subordonné. Cet autre point de vue, dont la seule nature est apparente, c'est : celui du noir pour le blanc, du travailleur manuel pour le travailleur intellectuel, bureaucrate syndicaliste ou partisan, celui du malade pour le spécialiste de la santé, celui de la femme pour l'homme . . . bref, toujours celui de l'autre pour l'un. Cet autre point de vue surgit donc de ce continent noir inexploré, de cette société hors-la-loi qui existe partout, fonctionne efficacement, et dont l'aménagement dia-logique précède déjà tous les modèles prétendus l'améliorer...

Il s'agit de rompre avec la tyrannie du logocentrisme ${ }^{4}$ pour redonner toute son importance à l'analyse des "stratégies " ${ }^{5}$, qu'il ne faut pas hésiter à qualifier de vitales, déployées entre les protagonistes lors de l'énonciation de leurs actes de langage. Du fait que toute proposition s'articule nécessairement sur ces stratégies alternatives, jamais univoques, mais, de par leur alternance même, multiplement systématiques et logiques, nous inclinons à comprendre le processus interdiscursif comme le lieu communicatoire d'un "échange " de paroles et d'actions. Lieu purement tractatif où chacun des partenaires en présence (individus, groupes ....) envisagés réciproquement irréductibles, constitue à la fois son autonomie et sa dépendance. Que l'on

4. «On connaît la démarche la plus radicale pour sortir des réseaux de la rationalité logocentrique : elle consisterait à lui substituer son autre. C'est la pensée marxiste de la production comme pratique destructrice du verbalisme, du travail comme effraction effective de la clôture culturelle. "F. Jacques in Dialogiques, recherches logiques sur le dialogue, Paris, Puf. 1979 , p. 390.

5. "L'appareil de l'énonciation ", au sens de E. Benvéniste : "L'appareil formel de l'énonciation ", Langages. XVII, Paris 1970. 
pourrait appeler encore l'élaboration commune de son " moi » avec les autres. Ce qui revient à dire que, si la connaissance vraie de l'autre reste pour nous problématique, en revanche, on rétablit très solidement la communication, et même, on constate qu'on ne peut que communiquer, qu'échanger ${ }^{6}$, c'est-à-dire : négocier.

\section{POSITION THÉORIQUE DU PROBLÈME DE L'ALTÉRITÉ.}

$\mathrm{Du}$ solipsisme invincible au collectivisme dérisoire, de l'aporie à la fiction, du paradoxe à la dogmatique déclarée, le problème de l'autre connaît diverses formulations philosophiques. Résumons-les schématiquement en utilisant partiellement, mais en l'élargissant à nos dimensions épistémo-éthiques, l'analyse de F. Jacques ${ }^{7}$.

\section{1.1 Le problème théorique n'existe pas (au plan philoso- phique).}

\subsection{Il est sans solution.}

6. Cf. avec K.O. Apel, Fondation ultime de la raison, in rev. Critique, Oct. 81, no. 413, p. 928. " La pertinence d'une fondation philosophique ultime de la raison réside alors dans un argument réflexif : on ne peut décider au niveau du discours et de la pratique ni pour ni contre les règles du jeu du langage transcendantal sans avoir déjà présupposé ces règles. "

7. F. Jacques, op. cit. Notre perspective s'écarte de celle de F. Jacques en ce que celui-ci pose sa problématique dialogique dans "l'a-priori »'de « la fondation transcendantale " (p. 392), cherchant à " cerner les règles universelles d'une compétence pragmatique " (p. 391). Ce qui oblige à une décision épistémologique au moins aussi arbitraire que celle qu'il reproche à Saussure : " il nous faut établir la médiation d'une activité communicationnelle (laquelle reprend selon nous la place exacte de la vérité transcendantale) et trouver ce qu'au juste on peut en dire a-priori " (p. 391-92). Ainsi, la pragmatique de Jacques au lieu d'être une ouverture décisive sur un champ de questionnement (éthique) limité mais applicable aux cas régionalement traités, demeure une problématique de questions générales non moins a-priori que l'axiomatique des questions générales qu'elle tente de fonder. "Si la position constituante des esquisses catégoriales de l'objet comporte un aspect axiomatique, celui-ci n'est que l'envers d'un aspect pragmatique et laborieux » (p. 392). Bref, il s'agit d'un retournement épistémologique au sens stratégique, c.-à-d. " cataphorique " du terme. Le paradoxe veut que l'un ne puisse se substituer à l'autre qu'en prenant sa place... Néanmoins, la rigueur et la précision de ce travail d'envergure, à notre connaissance le plus important sur cette question, en font sans doute l'un des meilleurs instruments conceptuels en référence à la recherche en pragmatique du langage.

Addenda :

Entre le moment de la rédaction et celui de la publication de cet article, ma recherche a évolué, dans le même sens de la différence, marquée de respect, pour la pensée de F. Jacques. Ce sont désormais les termes : diathèse, diatbésis et diathétique, qui élargissent les contenus travaillés ici autour de la notion de dia-logie. 
1.3 L'existence des "other minds" renvoie à une croyance purement instinctive (C'est le point de vue d'un B. Russell. $)^{8}$

II 2.1 Le problème théorique n'a aucun sens (comme absurde).

2.2 Quoi qu'il puisse en être, sa formulation reste impossible.

2.3 Pour Descartes et les pensées du cogito, où le sujet est posé dans un rapport privilégié avec lui-même, la connaissance de l'autre en tant qu'autre (sujet personnel jouissant, réciproquement, d'un statut ontologique équivalent) est inaccessible à la pensée objective comme à l'analyse réflexive.

2.4 La connaissance de l'autre reste d'ordre analogique ; sa modalité ramène au probable.

2.5 Il apparaît impossible de s'assurer d'autrui dans l'ordre des raisons d'un sujet connaissant, au même niveau où ce dernier s'assure ordinairement de lui-même, des objets physiques ou intellectuels de l'univers qui l'entoure, voire même de Dieu aux sens philosophiques du terme. F. Jacques remarque que, de ce point de vue : «Le proche ne surgit pour moi qu'à l'occasion d'un sentiment ou d'une passion qui n'a pas reçu de statut philosophique. Soit encore qu'objet d'application d'une règle universelle, il n'appelle aucune remarque spéciale $"$ ?

III 3.1 Le problème théorique a une position philosophique. Celle-ci est d'ordre pratique, au sens qu'une philosophie pratique kantienne suppose à ce terme (ce qui est rendu possible par la volonté).

Remarque : Selon F. Jacques : "Kant ne traitera pas du problème comme de celui du monde extérieur, dans un projet spéculatif et selon les catégories de la nature, mais en fonction de l'intérêt pratique de la raison et selon les catégories de la

8. Cf. B. Russell, Problems of philosophy, Home University Library, 1912.

9. F. Jacques, ibid, p. 20. 
liberté ${ }^{10}$. Disons, toujours selon sa perspective, que Kant dépasse donc déjà le point de vue strictement substantif du sujet substantiel, au sens métaphysique, pour, en quelque sorte, inaugurer le point de vue «pronominal " du sujet volontaire. En suggérant malgré tout que : « Kant est plus attentif à la pureté de la bonne volonté »-qu'on se souvienne du mot de Péguy ${ }^{11}$ "qu'à l'existence même de l'autre $~^{12}$. F. Jacques souligne qu' : "Un tel acte ne correspond pas à une vérité connaissable " ${ }^{13}$.

3.2 Si l'acte pratique ne correspond effectivement pas à un ordre de connaissance vraie, on constate en revanche que cet acte est constitutif du soi et d'autrui en ce qu'on tend vers lui : 1) pour s'apercevoir soi-même, autant que : 2) pour apercevoir autrui que : 3) pour être aperçu de lui. L'analyse du rapport maître/esclave ou, législateur/sujet de la loi, montre que le législateur est celui qui constitue l'autre en l'aliénant, selon le désir-alibi de cet autre. C'est-à-dire en l'instituant sujet de sa loi, laquelle incarne pour chacun son désir de constitution $^{14}$. F. Jacques s'accorde avec cette perspective lorsqu'il écrit que : "cet acte (la relation volontaire) la constitue (la personne qui le pose) comme sujet pratique, en même temps que son corrélat. Que l'acte s'achève, que le sujet se replie, et tous deux à la limite cessent d'exister $"{ }^{15}$

Remarque : On voit immédiatement la transformation du problème qu'opère un tel élargissement de la relation intersubjective (applicable selon nous à, intercollective) par cette notion de relation constitutive ou encore, au plan phénoménologique, de « coexistentialité ». C'est cè qui apparaît dès qu'il est admis que l'existence d'autrui ne soit pas « un pur roman . . . » selon le mot de Sartre. En d'autres termes, d'après le même auteur : «quand l'autre est celui qui me voit et non plus celui que je

\footnotetext{
10. F. Jacques, ibid, p. 21.

11. "La morale de Kant a les mains pures, mais elle n'a pas de mains ", dit en substance la formule complète.

12. F. Jacques, ibid, p. 21.

13. F. Jacques, ibid, p. 21. (N.B. c. -à-d. vérité connaissable au sens logico-mathématique).

14. L'hypothèse de l'aspect constitutif de ce rapport a déjà fait l'objet d'une étude de notre part in : La cataphore, ou l'écriture politique du sens, Phi Zéro, dec. 1981.

15. F. Jacques, ibid, p. 21.
} 
regarde, mon être-devant-autrui constitue précisément l'expérience cherchée ». Cette expérience qui constitue l'un et l'autre l'un par l'autre, c'est là ce que nous entendons par coexistentialité, ce que nous nommons une distribution dia-logique des partenaires en présence (à savoir : 1) l'un 2) l'autre 3) l'environnement culturel et naturel - le « monde " à négocier en commun).

Penser le monde d'une part, et d'autre part, l'autre, comme n'importe quel objet de ce monde, en recourant à une classification objective quelconque c'est là chosifier cet autre et ce monde en prétendant leur assigner leur place (de femme dans le monde des hommes, c'est-à-dire pensé par et pour des hommes, par exemple) ${ }^{16}$.

Penser l'autre comme " altérité irréductible », en tant qu'il est nécessaire à ma propre constitution d'être pensé par cet autre (ou ce groupe). Seule l'acceptation de cette équivalence, comme "égal désir de tous " ${ }^{17}$ : penser/être pensé, réciproquement, peut m'éviter cette réification symétrique qu'autrui ne peut que logiquement m'imposer, dès que je prétends l'exclure comme objet, ou l'aliéner comme sujet de ma loi, de ma logique, ou de ma définition. Dès lors, penser le sujet féminin comme altérité radicalement nécessaire, transforme la position univoque et réductrice du regard masculin (son autre). Ce, en lui permettant d'envisager (dia) logiquement la puissance de ce sujet comme susceptible d'explorations interpersonnelles, ainsi que de confrontations positives et négatives, au sens politique le plus fort ; soit en ce qui concerne le trafic des enjeux de pouvoir. Ce type de relation dia-logique où l'autre (le sujet féminin), ne peut jamais être réduit au même (le sujet massculin), en ce qu'ils sont posés comme irréductibles selon une perspective de coexistentialité, n'intéresse pas seulement le théoricien travaillant sur les concepts d'égalité destinés à l'aménagement des rapports hommes/ femmes. Elle sous-tend, et ce n'est pas là son moindre avenir,

16. Ceci au nom d'un effet de vérité qui n'est rien d'autre que l'alibi d'une prise de pouvoir de l'un sur l'autre (Cette réification par objectivation trouve sa limite dans l'aporie insurmontable qu'entraîne sa radicalisation : si le monde n'est que ma représentation, quelle garantie que je ne me représente pas moi-même? Mon moi ne représentant peut-être qu'une continuité de mise en scène . . .). Cf. F.J. p. 41-42.

17. Selon la belle formule galthérienne . . . cf. Y. Gauthier, sém. rech. Syntaxe et sémantique du concept d'égalité, U.d.M. nov. 1981, (p. 7). 
un renouvellement possible de la formulation des rapports hommes/hommes (dominants/dominés, individu/groupe, etc.) ${ }^{18}$.

En effet, la distribution dia-logique peut-être posée comme s'appliquant non seulement en termes de philosophie pratique et d'éthique, mais, ainsi que $\mathrm{F}$. Jacques en fait la démonstration, en termes épistémologiques et logiques. S'agissant de catégories essentiellement pragmatiques, il ne serait peut-être pas abusif de parler de dia-logie comme d'une perspective épistémo-éthique. Puisque «moi », comme « autrui » n'apparaissent l'un par l'autre qu'au terme de l'accomplissement d'un acte, le plus généralement de parole, qui a immédiatement l'effet d'un contrat social (lequel n'a d'autre fondement que l'articulation de sa cohérence interne, en regard de la nécessité pour les partenaires de se constituer, réciproquement ; le plus efficacement possible).

\section{LA QUESTION DE L'ALTÉRITÉ.}

Si, comme il est admis ici, le cogito cartésien arrive à dégager l'ego de l'entreprise totalisante du dogmatisme traditionnel, c'est, nous semble-t-il, pour l'isoler irréversiblement dans la représentation monadique du sujet transcendantal ou du moi psychologique. Du même coup, la question de "l'autre " se trouve éludée : a) comme irreprésentable, b) son traitement facultatif, puisqu'informulable, c) ses intérêts éthiques et ses droits indéfendables d) toute cause comme vouée à l'improbable, à l'échec ou à la réduction à celle de l'un par l'autre. Ainsi, chez un Leibniz, où l'interrelation (subjective) des monades suppose la médiation purement logique d'un tiers ; en l'occurrence Dieu.

L'égologisme radical de l'humanisme classique ne saurait trouver égoïstement sa propre fin qu'en lui-même et sa médiation qu'hors de lui-même, c'est-à-dire «Tout-autrement». Impasse symétrique de son retournement, le collectivisme massificateur, en niant tout aspect personnel au « je » individuel, nie du même coup tout « autre », et surtout toute possibilité d'alternative (homme/femme), au seul profit d'un pur procès

18. Au rapport homme/homme qui, dans presque toutes les institutions, place inexorablement les partenaires dans un rapport œdipien d'exclusion, il sera épistémologiquement préféré le rapport homme/femme, plus spontanément significatif de la différence, que du même. 
sans sujet ni fin, suivant la formule consacrée par la dialectique marxiste orthodoxe.

Rousseau . . Max Sheller, Bergson semblent pouvoir être considérés comme les pères d'un altruisme moderne fondé intuitivement sur la bonne volonté. Celui-ci recherchant dans le désir d'autrui le transport d'une orientation absolue. Mais, toutefois, comme le note F. Jacques : « . . . incapable(s) de penser le rapport transitif entre les personnes autrement que par la fusion affective des ego ${ }^{19}$. On peut faire remarquer qu'un tel altruisme gratuit n'est que la transposition philosophique d'une pensée directrice essentielle au Judéo-Xto-Islamique dans son orientation éthique générale. Celle-ci ayant été systématiquement absorbée par les Loi, Torah, Shariah d'une part, et d'autre part, par les diverses spéculations théologiques du Tout-Autre. Ce Tout-Autre dont la vérité évapore " autrui » devant " moi ", me le cache en ce que cette fascination métaphysique m'empêche de voir le monde et, au cœur de ce monde : l'autre. C'est là le Dieu-alibi, l'idole dont F. Nietzsche proclamait (et réclamait) la mort, comme préalable à toute discussion de l'homme sur l'homme. À la constitution de l'un par l'autre, hommes et femmes, pourrait-on ajouter.

C'est pour cette raison, croyons-nous, que, "l'autre » naturel de l'homme, son partenaire exact (au sens darwinien), a vu sa présence être traditionnellement niée " au nom »du Tout-Autre-alibi. Dans l'histoire, la femme a systématiquement été exclue comme force maligne ou "sorcière », c'est-à-dire comme rivale absolue de Dieu et de l'Ordre, identifiés au même. Celle qui oppose son irréductible présence à l'absence (transcendantale ou immanente) du Dieu-régisseur-et-maître de la certitude des certitudes : la mort. Tout «Ordre du monde ", toute "Vérité », réclamant une telle stabilité, que lui refusera toujours l'imprévisible dialogue, l'interminable négociation du quotidien avec l'éphémère autrui.

Or cette négation, pour avoir systématiquement travaillé la culture dans son ensemble, n'est-elle pas pour une grande part responsable d'avoir empêché un rapport homme/femme de

19. F. Jacques, ibid, p. 23. 
s'établir institutionnellement sur le mode dia-logique (hormis, bien sûr, en ce qui concerne le registre hors-la-loi des relations amoureuses) ? Et n'est-ce pas justement en instituant les hommes et les femmes dans une relation hiérarchique au lieu de favoriser leur rapport colloquial naturel ? Sinon, comment comprendre qu'à peine l'alibi ou Tout-Autre effacé, la femme apparaisse au monde comme Dieu lui-même ne l'a jamais été (qu'une seule fois peut-être !).

\section{**}

Un mot sur la pensée de Hegel dans laquelle « l'autre » se dégage comme : celui qui me fait face, et qui, en vertu de son devenir ne peut pas être inclus dans la totalité de l'être exprimé. Il s'échappe ainsi cas par cas et, par là, menace cette expression (c'est-à-dire l'édification rationnelle de cette expression, en ce qu'il peut indéfiniment la renouveler). L'autre reste donc celui avec lequel « je " dois négocier cette expression. Le seul aspect nouveau et qui intéresse notre perspective, on le devine, c'est cette mise en évidence de la tractation du pouvoir, la nécessité de négocier. En d'autres termes l'introduction à une philosophie du Droit.

Si donc l'existence d'autrui échappe au roman que nous pouvons nous en faire, la question surgit : quels sont ses droits ? et quelle est la portée de l'expression (lois, contrats . . .) qui concerne ces droits ? enfin, qui (de « lui » ou de " moi ») déterminera cette définition qui « nous» concerne réciproquement, et comment (méthode, logique . . . ) délimiter la portée de cette expression?

L'univocité du positionnement hiérarchique tel qu'il se réalise concrètement dans le rapport : dominant/dominé traditionnel, ne peut qu'empêcher cette négociation d'avoir effectivement lieu, puisqu'il se fonde sur la rature (exclusion) ou l'aliénation (asservissement) de l'un par l'autre. Le principe de ce rapport consistant simplement à empêcher l'alternance des partenaires $(a>b, b>a)$ de se produire. Cette alternance interdite par la loi, les tabous, les habitudes . . . est maintenue fermement en place par le réseau institutionnel nécessaire à son application (chef de famille, bureaucratie, armée . . .) qui médiatise cette relation. 
Le problème des consciences « médiées » ou médiatisées, c'est que l'effacement des catégories subjectives s'effectue au profit unique de celui, ceux, qui effectuent la médiation et, préalablement l'imposent par un seul principe (exclusion de tous les autres) « philosophique ", dont le rôle se borne à justifier (articuler rationnellement), la nécessité-alibi que la médiation soit le fait du tiers qui la pose. La métaphore juridique est évidente. Ce tiers instructeur du procès, et qui maîtrise les catégories du jeu de société, n'est autre que le même (son délégué), que le législateur, qui en a posé les règles. En prétextant, à bon droit d'ailleurs, qu'il représente l'unique solution pour surmonter un face à face intolérablement monadique entre des partenaires préalablement interdits de parole.

Comment, donc, surmonter cet impossible face à face de sujets, soit transcendés soit transcendantaux, où l'autre est inexorablement exclu, réifié ou aliéné ${ }^{20}$ ? Cela, tout en préservant l'expérience de proximité qui, à la fois découvre et se fonde sur l'irrépressible intimité de la personne, afin de ne pas diluer la constitution essentielle, individuante, d'autrui dans une généralité dérisoirement abstraite - et dangeureusement sujette à l'annexion?

Les contours d'une réponse apparaissent, liés à la critique de l'interdiction qui frappe le principe d'alternance de la parole échangée par les partenaires du jeu social. Aussi bien dans les relations homme/femme que dans les relations homme/homme d'ailleurs.

\section{LA POSITION DIA-LOGIQUE.}

Nous sommes conscients que l'ouverture de ce dossier n'est pas une mince affaire. Qui ne voit en effet qu'une critique de l'interdiction qui frappe le principe d'alternance est liée, au plan épistémologique, à une remise en cause du principe de noncontradiction, de raison suffisante et nécessaire etc. ? en ce qu'il suppose justement l'univocité et l'exclusion comme règles absolues. Or, si on ne lui déniera pas une sphère mathématiquement

20. «La recherche classique de l'être peut admettre la transcendance ou l'immanence, non la proximité (le proche). Elle autorise l'universalité du savoir, non la singularité des existants personnels". Ibid, p. 18. 
idéale d'applications purement formelles, il n'en sera pas de même en ce qui concerne certaines prétentions d'utilisations illimitées; tout particulièrement dans les champs éthique et politique $^{21}$.

Précisons encore qu'une critique comme la nôtre n'est qu'indirectement préoccupée de savoir si l'on peut ou non «. . formaliser les sciences humaines ${ }^{22}$. $\mathrm{Ni}$ de trancher la question en faveur ou en défaveur d'un humanisme effectivement dépassé. Notre préoccupation vise à distinguer entre chaque construction théorique et l'utilisation politique qui peut en être abusivement faite, pour servir «cataphoriquement " d'alibi logique au principe d'exclusion-annexion; lequel fonde réellement le pouvoir des définisseurs.

Articulée sur le principe d'alternance - en fait tripartite - des partenaires et de leur environnement, peut-on dégager du rapport logico-linguistique une logique confrontative dialogique? Une logique ni fondationnelle ni universalisante mais, simple outil intellectuel, une articulation distributive non transcendantale indéfiniment disponible pour assurer la meilleure performance aux conditions d'un échange allocutif/délocutif ? "Nous opérons ensemble dans le travail de la preuve, écrit Francis Jacques, dans un monde qui nous est commun par notre collaboration même. Dès lors, je pressens qu'autrui me fait face comme celui à qui j'exprime tout ce que j'exprime. Le seul corps qui conditionne de manière nécessaire et suffisante ma perception d'autrui, c'est le corps des mots et des actes de langage où s'incarne la parole $"{ }^{23}$.

Cette logique confrontative, cette dia-logique s'appuie donc avec force sur ce rapport que, l'un et l'autre, co-existent au sein d'un monde commun co-référentiel. Nous voyons ici se dessiner le rôle du troisième terme de notre articulation : la confrontation au monde. Monde, pluralité tramée de lieux physique, technique, politique, imaginaire, etc. ; pluralité temporelle de superpo-

21. En philosophie du droit, par exemple, la critique des aspects syllogistiques du droit romain, en cherchant à limiter leur portée, tout en acceptant une utilisation partielle nous semble aller dans ce sens. Cf. J.J. Salvail, Théorie du droit ou doctrine informe?, Phi Zéro, avril 1981.

22. Cf. Y. Gauthier in Chercheur, vol. VI, no. 4, octobre 1980.

23. F. Jacques, ibid, p. 27. 
sitions impossible à hiérarchiser au réel de l'histoire du phénomène humain ; monde, pluralité qu'appelle toute topologie communicationnelle, ainsi que l'a pressentie avec insistance la modernité depuis l'exigence phénoménologique de Husserl.

Ainsi, selon nous, "l'autre " condense en lui ce double visage : a) de la personne d'autrui et, b) du monde (vécu dans telle société particulière), qui à la fois nous divise, en nous espaçant dans la durée, et nous réunit comme liés au même univers de référence. Dès lors, ce troisième terme admis à titre co-référentiel pourra être lui aussi envisagé comme irréductible, et non pas seulement (mais aussi) comme simple représentation au sens métaphysique, ou comme simple production, aux sens marxiste ou constructiviste de ces termes. Ce n'est plus la Vérité d'une connaissance ${ }^{24}$, dont le pouvoir-alibi cherche systématiquement à se masquer, qui nous intéresse ici, c'est le travail commun de la preuve qui désormais retient notre attention. C'est l'établissement d'un réseau de signification (l'usage) dont la cohérence soit susceptible d'utiliser toutes les ressources de la rationalité, de l'intuition, de la logique ou de l'imaginaire, et de l'un et de l'autre. Pour déployer, non un Ordre universel et totalisant mais une organisation pour nous, partielle, temporaire et collective, où je (femme, par exemple) puisse devenir autre, dans le monde actuel des autres (hommes) et réciproquement.

Et il semble bien, malgré la « décision épistémologique » de F. de Saussure selon la forte expression de F. Jacques, qu'une telle structure d'opposition confrontative et alternative soit partie intégrante de la logique, de la langue elle-même, et la travaille de l'intérieur. Selon nous, l'hypothèse de l'« inversibilité " dialogale n'est pas qu'un désir, elle théorise le fait de parler. « Je » devient « Tu », l'ego devient l'autre, et inversement avec une fluidité et une solidité telles que : «Tout se passe comme

24. "Avoir contribué à casser une vérité intangible et éternelle n'est peut-être pas l'un des moindres titres de gloire de la démarche scientifique " écrit le biologiste F. Jacob, op. cit., p. 13. On se souvient que la question de M. Foucault (in Les mots et les choses) qui demande s'il peut exister un Savoir sans vérité. Or le court-circuitage systématique, que le jeu allocurif / délocutif gouverné par le principe d'alternance opère sur la discursivité, nous semble correspondre assez bien au discontinuisme prôné par ses théories ; aux plans éthique et politique, du moins. La discursivité devenant interdiscursivité, la continuité n'a pas besoin d'être fondée idéologiquement (comme chez M.F.) ; en deça de la règle, elle est le jeu lui-même. 
si le langage avait résolu d'un seul coup les deux problèmes de la communication et de la subjectivité ", remarque subtilement l'auteur précité, et il ajoute : « Chacun, en assumant l'indicateur « je » se déclare comme personne unique, cependant qu'il s'approprie le système des signes et convertit le langage en énoncés discrets ou énonciations ${ }^{25}$. Il suffit de faire ressortir que ce que dénote le langage, c'est précisément le «monde » en question. Et, que si « je » s'empare, même univoquement du système d'énonciation, ce n'est pas seulement pour émettre, mais encore pour être reçu. Or pour savoir si, et comment, l'émission a été perçue, il faut nécessairement faire jouer une structure d'alternance ; c'est-à-dire, que la bouche émettrice se positionne à son tour comme oreille réceptrice et que l'oreille d'en face prenne position de bouche.

Le dialogue (dia-logos) se présente ainsi comme structure : a) d'affirmation d'au moins deux logos (parole), de mise en relief et d'accentuation de, b) leur différence irréductible, c) d'alternance de leur position, qui imprime une irrésistible bifurcation à l'interminable négociation où se joue le «libre " trafic du pouvoir ; du moins si ne cesse jamais son va-et-vient de l'un à l'autre. Ce, à propos 1) des intérêts de l'un, 2) de ceux de l'autre, 3) du rapport qui les unit au monde qui leur est commun.

Remarque : Le jeu du pouvoir-trafic, ininterrompu, s'oppose au pouvoir-exclusion-annexion tel que défini par la philosophie de Platon à Hegel, critiqué et déconstruit par Marx, Nietzsche, Freud, Bataille . . . et toute la modernité derrière eux, selon diverses perspectives et au nom d'intérêts souvent divergents. L'enjeu d'un tel pouvoir-exclusion ? Supprimer l'inter-locuteur, l'émetteur potentiel, lui fermer la bouche, au nom de l'univocité, en lui "enseignant " la répétition de la même vérité ; afin de mieux l'accaparer comme oreille, de l'annexer comme puissance réceptive, femelle, territoriale . . .

L'alternative dia-logique se pose, comme une ouverture irrépressible dans la capitalisation totalisante opérée par le désir

25. Cf. F. Jacques, ibid, p. 36. 
de l'ego, cherchant à unifier et à massifier "l'autre », tous les autres, en-soi et pour-soi. Bien au delà d'une fiction de mauvais grammairien apparaît ici, dans cette brèche même qui permet le recul d'une échappée, la réalité perverse de cet exclusivisme inassouvissable justifiant "l'un " de supprimer ou d'annexer tous « ses" autres ${ }^{26}$.

\section{$*^{*} *$}

Aussi, vue de cette brèche, la révolution féminine nous apparaît-elle comme l'une des voies privilégiées placées au carrefour d'un dépassement vital de cet excès d'Ordre ; orientée elle aussi vers l'aube d'un nouvel âge, de raison (?), étrangement imprévisible.

Privés de tous les "ciels", de tous les dieux, de toutes les vérités et de toutes leurs béquilles ipsotropes ou ipsofuges, ne s'agit-il pas en définitive de négocier l'incoercible peur de se retrouver face à face ? Ensemble, plongés dans l'inconnu de vivre, non une représentation u-topique, mais ce monde-ci, cette société là, pour ce temps qui passe, le nôtre ?

Remarque : L'étrange exclusivisme du discours féministe tente de jouer une formulation à la limite même où la rationalité "phallogocentrique " ${ }^{27}$ impose institutionnellement son autorité sous l'apparence d'un discours de vérité ; et ce, sur le mode de la substitution pure et simple. S'il persiste dans cette voie, selon nous, il réduira à rien son absolue différence. Il n'en reste pas moins que, pour le temps qui nous occupe, dissonance dans le bel édifice de l'Ordre masculin, ce discours le traverse et l'ébranle comme un $\mathrm{cri}^{28}$ !

Par delà le simple questionnement épistémologique, cette interpellation s'avère une problématique majeure, qui trahit, jusque dans l'incohérence de sa mise en forme, l'une des plus

26. Peut-être n'est-il pas déplacé de voir là, dans l'irréalisable de ce désir tyrannique, l'ingrédient traumatique de cette angoisse propre à la conscience malheureuse, tel qu'analysé, parfois avec un excès de morbidité, par l'existentialisme contemporain.

27. Selon le mor de Claude Lévesque. Cf. descriptif du projet de Recherche sur les théories de l'égalité, sem. rech., U.d.M., octobre 1981.

28. ... . et non comme un phantasme. Sur la dynamique du cri, cf. l'article de C. Lévesque, Dissonance, in Études Françaises, février 82. 
puissantes tentatives collectives de notre temps d'échapper à l'enfermement de l'énonciation univocifiante. Dans une perspective dia-logique ce discours apparaît comme la couverture superficielle d'un enjeu de signification absolument vital. Celui, proprement politique, de la conquête du pouvoir correspondant aux espaces culturels particuliers, soit typiquement féminins ou masculins, soit négociables en commun. Mais dont la mainmise se trouve à l'heure actuelle incontestablement à l'avantage du seul définisseur : masculin.

Instrument de négociation par excellence, la perspective dia-logique (si l'on s'ouvre à sa qualité d'hypothèse de travail) pourrait stimuler la formulation d'un outillage conceptuel d'importance, susceptible d'assurer au plan éthique, à la fois l'autonomie relative (en relation) de la personne et sa solidarité coexistentielle avec son environnement sociétal ; et même écologique au sens général. $\mathrm{Ce}$, du fait que cette perspective doit permettre de négocier non seulement l'espace culturel attribué d'emblée à chaque partenaire, mais encore les règles qui définissent ces espaces (ce qui, on le voit, renvoit immédiatement à un projet pédagogique).

Le désir d'émancipation des femmes, articulé sur un tel cadre théorique, devrait donc, logiquement, s' " ensoucher " dans un projet de signification plus fondamental, actif, de définitions épistémo-éthiques et d'attributions politiques des espaces culturels qui leurs sont propres. Soit en deux mots leur permettre de revendiquer puis de conquérir la compétence juridique de formuler des lois concrètes, pour un sujet légal féminin qu'il leur appartient à elles seules de typifier selon leurs définitions. Autrement dit, écrire la loi (commune) mais en tant que femmes. Devenir des définisseuses, pour se constituer par elles-mêmes.

Remarque : Pourquoi cette insistance sur la relation légale ? C'est qu'il nous semble qu'au stade actuel des États-nations modernes, formule politique au sein de laquelle a surgi la révolution féminine, mais qui risque de durer plus longtemps qu'elle, le rapport juridico-prescriptif s'avère être à la fois le plus puissant outil des réalisations socio-culturelles, en même temps que le 
plus immédiatement susceptible d'être concrètement conquis, « également " par des femmes et par des hommes.

Pourvu que ce passage du être constitué (être énoncé par la Loi, celle des hommes) au se constituer (énoncer la loi, non pas des femmes, mais en tant que et pour des femmes) sache tenir compte rationnellement de l'infranchissable réseau des déterminations de tous ordres. Enfin, que ce nouveau jeu colloquial, laisse place à l'aléatoire et à l'imprévisible des différences inhérentes à, ou privilégiées par, chacun des partenaires, inexorablement solitaires et solidaires du sens d'un monde affirmé ; commun. Et qu'importe si, d'avance on le sait, la négociation restera toujours difficile, quand ce n'est pas . . impossible.

L'essentiel n'est-il pas : accepter d'entrer dans le jeu dialogique de l'alternance, cherchant par là à promouvoir dans "l'autre " l'irréductible de mon propre inachèvement, comme le sous-ensemble $e^{29}$ étrange sans lequel « je » n'existe même pas?

Département de philosophie Université de Montréal

29. Au sens de Michel SERRES, c.-à-d. : « un sous-ensemble étrange, c'est ce sans quoi le système n'aura pas lieu. "Et encore : « ce qu'un sous-ensemble prépare dans un système donné, c'est une bifurcation inattendue. "In conférence sur Les Anges, U.d.M., mars 1982. C'est précisément ce pourquoi nous constatons que, sans un principe d'alternance, toute stratégie communicative mène inexorablement à l'exclusion $d u$ destinataire. 